\title{
Inteligencia competitiva: corpus teórico y prácticas
}

Competitive intelligence: theory and practice

\author{
Montserrat GARcía Alsina, Eva ORTOLL EsPINET \\ Estudios de Ciencias de la Información y de la Comunicación, Universitat Oberta de Catalunya, España, \\ \{mgarciaals|eortoll@uoc.edu\}
}

\begin{abstract}
Resumen
Este artículo tiene como objetivo presentar un estado de la cuestión del principal corpus teórico de la inteligencia competitiva (IC). La literatura actual está compuesta por trabajos realizados por profesionales y por académicos. Este hecho y su origen multidisciplinar están originando cierta dispersión de temas vinculados a los procesos de IC y a su organización, que requieren de cierta unificación. Se exponen las temáticas detectadas: terminología, organización de la función, descripción de los procesos, evaluación, modelos existentes, y factores facilitadores e inhibidores de las prácticas de IC. Por último, se señala la necesidad de seguir con trabajos empíricos que validen el constructo existente, y de profundizar en dos líneas de investigación: la integración de los marcos teóricos existentes y la identificación de los factores facilitadores e inhibidores de las prácticas.
\end{abstract}

Palabras clave: Inteligencia competitiva. Gestión de la información estratégica.

\section{Introducción}

La Inteligencia Competitiva (IC) es una actividad existente desde hace milenios, y derivada de influencias de inteligencia militar, económica, comercial y política (Garcia, 2011; Juhari y Stephens, 2006). Sus prácticas surgen como consecuencia de la necesidad de las organizaciones de reducir las incertidumbres del mercado, para lo que precisan capturar y gestionar información estratégica del entorno.

Inicialmente hubo predominio de trabajos referentes a las prácticas de IC, por lo que en la construcción del corpus teórico ha sido relevante la contribución del ámbito profesional, con enfoque prescriptivo predominante. No obstante, progresivamente el ámbito académico se ha ido incorporando al estudio de estas prácticas y hoy contamos con infinidad de trabajos procedentes de diversas disciplinas, con enfoque teórico, y diseño de un paradigma asociado a la disciplina.

A pesar del considerable volumen de trabajos describiendo prácticas de IC o teorizando sobre éstas, aún hay temas de debate que apuntan a

\begin{abstract}
The aim of this paper is to present a state of the art of competitive intelligence $(\mathrm{Cl})$. The literature is integrated by works written both by practitioners and academics. This fact and its multidisciplinary nature originate a scattering of subjects related to the processes of $\mathrm{Cl}$ and its organisation, that requires an integrated approach. The paper exposes the topics detected: terminology, function organisation, processes, evaluation, models, and enablers and inhibitors of $\mathrm{Cl}$ practices. Finally, ti is exposed that the need of continuing with empirical works that validate the construct, and lines of future research are suggested: the integration of the existent theoretical frameworks and the identification of the enablers and inhibitors of IC practices.
\end{abstract}

Keywords: Competitive intelligence. Strategic information management.

que la IC como disciplina aún está en proceso de formación (Fleisher, Wright y Tindale, 2007). En consecuencia, aún es preciso seguir trabajando en la construcción del corpus teórico.

Por ello, el objetivo de este artículo es presentar un estado del arte de la IC, así como también de algunos de los aspectos que conforman una disciplina. En lo que se refiere al primer aspecto -corpus teórico- presentamos las bases existentes para validar el constructo existente, y las líneas de investigación, que hemos detectado en la literatura. Respecto a las prácticas, referenciamos el avance que se ha hecho en distintos sectores y países. Por último, analizamos algunos de los aspectos identificativos de una disciplina: formación de grupos o redes de especialistas que constituyan una comunidad científica, el establecimiento de publicaciones que facilitan la formación de redes, la existencia de un paradigma común a la comunidad científica que permita compartir las prácticas, definir problemas y legitimar métodos de investigación (Vanderstraeten, 2010; Kuhn, 2006) 
El resto del artículo está estructurado como sigue. Presentamos la metodología empleada, exponemos los resultados, y, concluimos con unos apuntes sobre los temas de discusión y futuras líneas de investigación.

\section{Metodología}

Hemos identificado la producción en el campo de la inteligencia competitiva a partir de las búsquedas, en distintas bases de datos: ISI web of knowledge; Business Source Complete (EBSCO); Emerald Journals Services; Elservier, Sciencedirect; Blackwell; Springer; IngentaConnect; Sage; Jstor; Lisa; Dissertation Abstracts; TESEO; TDX (Tesis Doctorals en Xarxa); EconLit; ISOC - Humanidades y Ciencias Sociales (CSIC); y Google académico.

La acotación temporal de las búsquedas fue de 2002 a 2010, tomando como precedente el estado del arte producido por Bergeron y Hiller (2002). Posteriormente otros estados del arte localizados contribuyeron a contrastar el avance del estudio (Dishman, Fleisher y Knip, 2003; Fleisher, Knip y Dishman, 2003; Knip, Dishman y Fleisher, 2003; Fleisher, Wright y Tindale, 2007).

Las palabras claves empleadas fueron las identificadas en artículos relevantes sobre el tema, y realizamos las búsquedas empleando términos en los idiomas de las bases de datos: inglés, francés o español. Concretamente: competitive intelligence, business intelligence; strategic information; business information; environment scanning; stakeholders information; corporate information; information analysis; patents information; intelligence agents; veille technologique; social intelligence.

El gestor bibliográfico Refworks facilitó la detección de duplicados, y el listado de publicaciones existentes vinculadas con la inteligencia competitiva, que a su vez nos permitía identificar dos aspectos: a) distintas disciplinas involucradas en el campo de la IC, y b) vías de comunicación entre los expertos que trabajan en IC. A partir de los resúmenes, seleccionamos los artículos más representativos para analizar con más detenimiento las temáticas tratadas, metodologías empleadas y líneas de investigación. Tras su lectura fueron emergiendo temáticas consensuadas en el grupo de investigación.

\section{Resultados}

Las temáticas que han surgido en el proceso de la investigación son las siguientes: términos y conceptos, génesis de la IC, orientación táctica o estratégica, actuaciones proactivas o reacti- vas, señales débiles, procesos de inteligencia, técnicas de análisis, herramientas tecnológicas, aprendizaje y gestión del conocimiento, innovación, aspectos corporativos, contrainteligencia, co-opetition, inteligencia territorial, papel del estado, perfiles profesionales, y metodologías empleadas en los trabajos empíricos.

Estos temas los presentamos agrupados en los siguientes bloques: 1) terminología empleada y definiciones existentes; 2) prácticas de IC; 3) fundamentos teóricos; y 4) vías de comunicación entre expertos de IC.

\subsection{Terminología y definiciones}

Hay diversidad de definiciones y términos referentes a IC para definir funciones y procesos similares (Brody, 2008; Liu y Wang, 2008; Pirttimäki, 2007; GIA, 2005; Hirvensalo, 2004; Bergeron y Hiller, 2002).

Términos empleados son: vigilancia (veille) seguido de varios adjetivos, dependiendo de la orientación y de los objetivos que tenga fijados la recogida de información (por ejemplo, vigilancia tecnológica o veille technologique, vigilancia comercial o veille commerciale; vigilancia de la competencia o veille concurrentielle, etc.), exploración del entorno (environmental scanning), inteligencia competitiva (competitive intelligence), inteligencia de mercado (market intelligence), marketing intelligence, inteligencia del competidor (competitor intelligence), inteligencia económica (intelligence économique), inteligencia del negocio (business intelligence), inteligencia territorial (intelligence territoriale), e inteligencia social (social intelligence). En los últimos diez años, hay artículos que además de IC como palabra clave emplean otros como Forecasting, Foresight, y por extensión Technological Foresight o forecasting, y en menor medida Future Studies (Garcia, 2011).

Una comparativa entre algunas de las definiciones que encierran estos términos permite ver la sinonimia de los términos, y la evolución que su significado ha tenido a lo largo del tiempo. De manera generalizada podemos concluir las siguientes similitudes y diferencias, y las preferencias de uso tal y como son ahora aceptadas en la literatura actual.

- Vigilancia, environmental scanning e inteligencia competitiva son sinónimos, aunque algunos autores insisten en su diferenciación (Garcia, 2011; Larivet 2009; Escorsa y Maspons, 2001). La preferencia en el uso de uno u otro término responde más a tradiciones históricas y culturales, puesto que hacen referencias a prácticas similares. Las diferen- 
cias en las prácticas se deben al grado de desarrollo de éstas en las organizaciones.

- Inteligencia competitiva es un término más amplio que los otros términos asociados a inteligencia (marketing intelligence, market intelligence y competitor intelligence). Por tanto, en ningún caso se deben emplear éstos como sinónimos. El uso de un término u otro tendría que ser para diferenciar o hacer hincapié en el aspecto al que está orientada la función de inteligencia, y, por tanto, sobre el que se busca información, y hacia donde se quiere dirigir la inteligencia generada.

- Inteligencia económica e inteligencia competitiva son sinónimos. La diferencia principal radica en la preferencia de uso en los países francófonos del primer término.

- Inteligencia de negocio o business intelligence $(\mathrm{BI})$, es un término que, con un origen en el ámbito de gestión del entorno, ha evolucionado y se usa con significados distintos. Actualmente se está empleando en dos sentidos. Uno, más restrictivo, para referirse a las aplicaciones que gestionan y explotan los datos internos de la organización, y que tienen como objetivo ayudar a la toma de decisiones. El otro desde un punto de vista más amplio, y como sinónimo de IC, para referirse a los procesos, a la tecnología, y a la gestión de información tanto interna como externa. Según un estudio hecho por GIA (2007), $\mathrm{Bl}$ es de uso más común en el ámbito profesional, seguido de inteligencia de mercado y en tercer lugar inteligencia competitiva. Otros estudios también indican preferencia de uso en Finlandia y Países Bajos (GIA, 2007; Pirttimäki, 2007).

- Inteligencia territorial es un tema que ha emergido en Francia en los años 90, y se utiliza para nombrar la función y el proceso de inteligencia llevado a cabo por las administraciones públicas a escala local, regional o estatal. El objetivo es, mediante el uso de la información, conocer el territorio y sus recursos, para crear riqueza y planificar políticas de desarrollo y sostenibilidad (Herbaux y Bertacchini, 2007; Bertacchini y Girardot 2006;). En la misma línea Alaqui (2010) considera la IT un proceso de intercambio de información estratégica a nivel local, y, además, incluye en la definición acciones propias de la Inteligencia Competitiva y de la Gestión del Conocimiento para impulsar el desarrollo de organizaciones y empresas que pertenecen a una misma área geográfica.
- Inteligencia social (Choo, 2002; Cronin y Davenport, 1993) e inteligencia pública (Solberg Søilen, 2005) son términos con menor uso en la acepción de gestión de información estratégica procedente del entorno. De las definiciones existentes, detrás está el papel otorgado a los estados para crear ventaja competitiva en su país. Este hecho permite señalar equivalencias entre el término de inteligencia social empleado en la literatura anglosajona, y el término de inteligencia territorial empleado en la literatura francófona, como algunos autores ya lo han hecho (Bourret, 2009). Por tanto, inteligencia territorial, inteligencia pública e inteligencia social son términos sinónimos, aunque el primero es el predominante para referirse a la gestión estratégica de la información, hecha por parte de los gobiernos o instituciones públicas y privadas para fomentar el desarrollo territorial.

Al margen de los términos empleados, todos incluyen los conceptos de recoger información sobre el entorno, almacenarla, analizarla, diseminarla y aplicarla para diseñar cursos de acción. La diferencias están vinculadas a los diferentes grados de aplicación de las prácticas, y a la amplitud temática objeto de las búsquedas de información.

En definitiva, los términos que parecen arraigar más son: inteligencia competitiva, inteligencia económica, inteligencia territorial y business intelligence en las acepciones antes señaladas.

\subsection{Prácticas de IC y formación académica}

Las prácticas de IC han existido desde hace milenios, tanto en el sector público como en el privado, aunque en unos países más intensamente que en otros (Calof y Wright, 2008; Juhari y Stephens, 2006; Hedin, 2004; Ikeya e Ishikawa, 2001; Rouach y Santi, 2001). Estos tipos de prácticas han continuado a lo largo del siglo XIX y XX, impulsadas tanto desde las organizaciones públicas como privadas.

Los estados y organismos oficiales han tenido y tienen un papel destacado en la introducción de prácticas de inteligencia competitiva a nivel estatal o a nivel empresarial. En primer lugar encontramos servicios de vigilancia tecnológica y la creación de observatorios impulsados desde las Administraciones Públicas, encargados de suministrar información tanto tecnológica como económica a las empresas que lo pidan, con el objeto de fomentar el uso y la cultura de IC (Comai y Tena, 2005; Viviers, Saayman y Muller, 2005; Carayon, 2003; Calof y Skinner, 1999; Martre, 1994). En segundo lugar, la Ad- 
ministración crea organismos regionales de asesoramiento a empresas orientados a apoyar a las PYMES para implementar sistemas de inteligencia competitiva. Este papel del estado permite apoyar a la industria nacional mediante la recogida de datos, análisis y diseminación de inteligencia para la acción (Wagner, 2003; Bergeron, 2000). Francia, Japón, y Canadá son ejemplos destacados de países con tradición en acciones gubernamentales para impulsar y apoyar la IC en las empresas.

Actualmente encontramos prácticas de inteligencia competitiva en diferentes países impulsadas tanto por la Administración y los gobiernos como por el sector privado. A lo largo del siglo $X X$, de manera progresiva, se ha ido desarrollando una metodología orientada a capturar información del entorno y analizarla para la formulación de la estrategia de la organización y crear inteligencia para la toma de decisiones (Heppes y du Toit, 2009; Calof y Wright 2008; Michaeli 2006; De Pelsmacker, 2005; Bergeron y Hiller, 2002; Prescott 1999; Porter 1987; Fahey, King y Narayanan, 1981; Ansoff 1975; King y Cleland, 1974; Thomas, 1974; Aguilar, 1967). En la literatura hay predominio de prácticas en las grandes empresas (Jain, 1984; Diffenbach, 1983; Fahey, King y Narayanan, 1981), no obstante en los últimos años se ha manifestado interés por las prácticas de IC en las PYMES (Olamade et al. (2011) Smith, Wright y Pickton, 2010; Kamoun-Chouk (2007).

Las prácticas de las organizaciones sin ánimo de lucro también han sido objeto de estudio. Estas organizaciones también tienen obligaciones y necesidades organizativas de obtener información del entorno. En primer lugar, necesitan planificar teniendo en cuenta su entorno, cada vez más competitivo y dinámico, para lograr la sostenibilidad de sus acciones (Weerawardena et al. 2010; Jain y Singhvi, 1977). Además, deben rendir cuentas de sus actividades y de la gestión de sus fondos (Brouard, Larivet y Sakka, 2010; Fox y Brown, 1998). Dependen de su entorno para obtener recursos financieros y humanos de los gobiernos, y de sectores privados, bajo la forma de donaciones o subvenciones. Por último, mediante la creación de valor y la obtención de beneficios pueden continuar su actividad (Brouard, Larivet y Sakka, 2010).

El desarrollo de las prácticas ha originado, aunque lentamente la incorporación de la IC en el ámbito académico. Algunas universidades han incorporado la IC en su oferta formativa dentro de sus planes docentes o de grado o como parte de postgrados. Los países pioneros en el ámbito de la enseñanza han sido Suecia, desde la década de los 70 (Hedin, 2004), Estados Unidos, desde la década de los 80 (Bensoussan y Densham, 2004) y Canadá y Reino Unido, desde la década de los 90 (Larivet, 2007; Calof y Brouard, 2004). A partir del 2000 universidades de otros países han incorporado estudios de IC, ya sea como posgrados o como asignaturas de grado: Alemania (Michaeli, 2004, 2006), Australia (Bensoussan y Densham, 2004), Corea (Kim y Kim, 2004), España (Ortoll y Garcia, 2008; Comai y Millán, 2005), Finlandia (Hirvensalo, 2004), Francia (Portail de l'IE, 2010; Carayon, 2003), Lituania (Stankeviciute, Orožekauskas y Jucevicius, 2004), Reino Unido (Wright et al. (2004) y Sudáfrica (Viviers, Saayman y Muller, 2005; Viviers y Muller, 2004). En este momento cada universidad y país han tenido iniciativas individuales para ofrecer grados y posgrados donde incorporan esta disciplina, por lo que algunos autores han apuntado la conveniencia de un programa formativo de IC común en el ámbito académico (Parker, Nitse y Davey, 2008; Pearce, 1971).

\subsection{Fundamentos teóricos}

Los temas que constituyen el corpus teórico de las prácticas de inteligencia los resumimos en los siguientes aspectos: función, ciclo, factores facilitadores e inhibidores, evaluación de la IC.

\subsubsection{Organización de la función inteligencia}

La exploración del entorno, como función, para recoger información, detectar amenazas u oportunidades, en algunas organizaciones queda formalizada en el diseño de sistemas de observación del entorno, y en otros está reducida a una práctica informal.

La función queda caracterizada por los siguientes factores:

- La orientación de los sistemas. Según el tipo de información que se captura y que circula dentro de la organización para generar inteligencia, la función se orienta de acuerdo a tres perspectivas: a) las temáticas de las búsquedas de información, el nivel organizativo donde se aplica la inteligencia y el marco temporal de impacto de la inteligencia generada, a corto o largo plazo, inteligencia táctica o estratégica, orientación reactiva 0 proactiva; b) el tipo de información que circula entre la organización y la competencia (pasiva, ofensiva, defensiva o prospectiva, y colaborativa); c) la relación entre la inteligencia obtenida y el entorno (reactiva o proactiva) (orientación táctica o estratégica); y d) actitudes de los directicos (reactiva y proactiva) (Hair, 2007; Culver, 2006; Heinrichs y 
Lim, 2005; Dishman y Pearson, 2003; Bergeron y Hiller, 2002; Peyrot et al., 2002; Rouach y Santi, 2001; Gieskes, 2000; Cartwright et al., 1995; Ghoshal y Kim, 1986; Daft y Weick, 1984).

- La frecuencia de la observación del entorno varía de una organización a otra, dependiendo de los medios disponibles, de la cultura organizativa, de las necesidades informativas identificadas o de la percepción de incertidumbre del entorno. Cuando la frecuencia es continua, o está definida la periodicidad de las búsquedas, la exploración es sistemática y está orientada a prevenir incertidumbres futuras y anticipar problemas $u$ oportunidades potenciales (inteligencia proactiva). Cuando la frecuencia es irregular, el sistema de exploración y su análisis se activa de manera puntual para dar respuesta a necesidades de información concretas, para resolver un problema detectado (inteligencia reactiva) (Bergeron y Hiller, 2002; Rouach y Santi, 2001; Choo y Auster, 1993; Daft y Weick, 1984; Fahey, King y Narayanan, 1981; Aguilar, 1967).

- La concreción de la ubicación de la función es relevante tanto desde el punto de vista de la cultura organizativa, como desde el punto de vista de obtención de recursos, logro de objetivos y rendimiento. Tiene influencias en su eficacia, ya que la ubicación puede influir en la recogida y diseminación de inteligencia, $y$ en facilitar un equilibrio entre los requerimientos tácticos y estratégicos de la organización. Hay distintas fórmulas de ubicación: a) centralizada, b) descentralizada, c) en redes de inteligencia, d) recogida informal, y e) externalizada. Los criterios de selección de una u otra fórmula son diversos, y falta acuerdo sobre cuál es la más adecuada. La falta de una unidad encargada de la función de inteligencia, o la falta de coordinación entre unidades o personas dedicadas a la función de IC puede tener consecuencias en cuanto a duplicidad de datos y esfuerzos de recogida y diseminación de información, o menor recogida de información y análisis y uso de producto de inteligencia y servicios (Bergeron y Hiller, 2002; Miller, 2001; Lackman, Saban y Lasan, 2000; Simon, 1999).

- La gestión de la función se refiere a la definición de procesos y procedimientos de observación del entorno, la asignación de responsabilidades para impulsar la IC y gestionar de manera efectiva sus prácticas y la asignación de recursos (Heppes y du Toit, 2009; Saayman et al. 2008; Wright y Calof, 2006).

\subsubsection{Ciclo de inteligencia}

Los procesos para cumplir con la función de inteligencia conforman lo que se ha denominado ciclo de inteligencia: identificación de las necesidades, recogida de información, organización y almacenamiento de la función, análisis de la información, generación de inteligencia, y diseminación y uso de inteligencia. Esta temática ha sido ampliamente estudiada y hay consenso en la configuración del ciclo de inteligencia (Bose, 2008; Dishman y Calof, 2008; Saayman et al. 2008; McGonagle, 2007; Antia y Hesford, 2007; Fleisher y Bensoussan, 2008).

En la primera fase del ciclo, la detección de necesidades, son relevantes los procedimientos para recoger los temas sobre los cuales buscar información, e ir actualizando el inventario de las necesidades (Vuori, 2006; Choo, 2002).

En la fase de recogida de información los temas de interés son a) definición de procedimientos para localizar, actualizar y/o validar las fuentes empleadas; b) tipo de fuentes de información utilizadas, incluidas las redes sociales, criterios de selección y su relevancia para las necesidades detectas; y c) los canales de obtención de información empleados (formales o informales). Estos aspectos influyen la orientación de la función, proactiva o reactiva, táctica o estratégica (González, 2012; Bégin et al., 2007; Vuori, 2006; Kourteli, 2005; Marin y Poulter, 2004; Choo, 2002).

En la fase de organización y almacenamiento de la información los temas de interés son: a) los procedimientos integrados de gestión de la información existentes en todos los departamentos y unidades de gestión, y b) las tecnologías de soporte disponibles en la organización (Choo, 2002).

Los focos de atención en la fase de interpretación y análisis de la información son cuatro: a) los productos y servicios de información, con valor añadido, elaborados $u$ obtenidos disponibles en la organización; b) los canales de difusión de estos productos y servicios; c) los espacios y las estructuras dentro de la organización disponibles para compartir, interpretar y analizar información; y d) las técnicas de análisis para extraer inteligencia (Fleisher y Bensoussan, 2008; Marin y Poulter, 2004; McGonagle y Vella, 2003; Kotorov, 2001; Marín-Llanes, Carro y Espin, 2001; Fink y Schlake, 2000).

En la generación de inteligencia los aspectos considerados son dos: a) el tipo de estructuras responsables de la toma de decisiones y el modelo de toma de decisiones adoptado; y b) cuáles han sido las decisiones y las acciones que 
se han diseñado durante el proceso de adaptación, teniendo en cuenta la inteligencia generada (Choo, 2002).

Transversal a estos ciclos esta la fase de feedback, que permite aprender a partir de la misma practica de IC, ya que permite reorientar el mapa de necesidades, reajustar procedimientos o mejorar la planificación de inteligencia. Al mismo tiempo informa del valor que los directivos dan al trabajo hecho por los profesionales de la IC, a la inteligencia recogida por el sistema de inteligencia y a la efectividad de las actividades de inteligencia (Weiss, 2002; Babbar y Rai, 1993).

A pesar de este acuerdo en la configuración del ciclo, en los últimos diez años, se apuntan modelos de IC donde se quiere dar cabida a aspectos complementarios del ciclo clásico que ayuden a comprender las dinámicas de funcionamiento de las partes del ciclo y la eficiencia en la generación de inteligencia. Algunos ejemplos son: aprendizaje organizativo (Choo, 2002) generación eficiente de inteligencia (Jaworski, Macinnis y Kohli, 2002), interelación del ciclo y la función (Dishman y Calof, 2008; Saayman et al. 2008).

\subsubsection{Factores facilitadores e inhibidores}

Varios factores influyen las prácticas de la IC en las organizaciones. Algunos de éstos Correia y Wilson (2001) los clasifican en factores individuales y en factores organizativos. Choo (2001) identifica cuatro grupos de factores: externos, organizativos, informacionales y personales. Otros autores ven aspectos de la cultura nacional como facilitadores o inhibidores de las prácticas de IC (Knip, 2006; Miller, 2002; Ebrahimi, 2000; Hofstede, 2001), o características del sector donde trabaja la organización (Hesford, 2008; Kourteli, 2005).

A pesar de que todavía es necesaria más investigación empírica, destacamos los siguientes grupos de factores: influencia del tamaño de las organizaciones; influencia del sector en el que las organizaciones desarrollan su actividad; factores individuales (responsabilidad de las personas hacia la vigilancia del entorno, análisis y diseminación y frecuencia de oportunidades para contactar personas bien informadas y estar en contextos ricos en información); factores organizativos (condiciones que determinan el acceso y el uso de información a partir de infraestructuras y estructuras organizativas), e influencia de la cultura del país (Choo et al. 2008; Saayman et al., 2008; Hesford, 2008; Kourteli, 2005; Calof y Dishman, 2002; Jaworski, Macinnis y Kohli, 2002; Wright, Pickton y Callow,
2002; Correia y Wilson, 2001; Simon, 1999; Hofstede, 2001). En los últimos años emerge el tema de las competencias y comportamiento informacionales como un grupo de factores que influye la eficiencia de las prácticas de IC (Zhang et al. 2010; Frion, 2009; Frion y Samier, 2009).

\subsubsection{Evaluación de la IC en las organizaciones}

La literatura sobre IC, de manera mayoritaria, reconoce el beneficio obtenido por las organizaciones que aplican la IC (Buchda, 2007; Herring, 2007; Davison, 2001; Fleisher y Blenkhorn, 2001; Lönnqvist y Pirttimäki, 2006; Pirttimäki, Lönnqvist y Karjaluoto, 2006; Thomas, 2001; Kilmetz y Bridge, 1999). A pesar de este reconocimiento, faltan evidencias concluyentes de estos beneficios, al haber ciertas dificultades para determinar y medir el impacto de la IC en una organización (Buchda, 2007; Lönnqvist y Pirttimäki, 2006; Bergeron y Hiller 2002).

Aunque existe literatura sobre como medir la IC (Pirttimäki, Lönnqvist y Karjaluoto, 2006), escasea la literatura basada en investigación empírica y las prácticas generalizadas de medición y evaluación del rendimiento de la IC en las organizaciones (Blenkhorn y Fleisher; 2007; Buchda, 2007; Marin y Poulter, 2004; Pirttimäki, Lönnqvist y Karjaluoto, 2006).

En consecuencia, desde diferentes ámbitos, como el financiero y el de gestión de la calidad, un volumen considerable de literatura enfatiza la necesidad de construir modelos de medición del rendimiento de la IC, su eficiencia, su efectividad y su impacto en una organización (Antia y Hesford, 2007; Blenkhorn y Fleisher, 2007; Buchda, 2007; Pirttimäki, Lönnqvisti y Karjaluoto, 2006; Lönnqvisti y Pirttimäki, 2006; Viscount, 2002; Davison, 2001; Fleisher y Blenkhorn, 2001; Sawka, 2000; Kilmetz y Bridge, 1999; Dashman, 1998; Herring, 1996).

Hay distintos enfoques para medir los beneficios de IC (función, ciclo, o productos generados), y distintos modelos de indicadores. Los primeros datan de principios de los años 90, y surgieron de investigaciones empíricas basadas en entrevistas o encuestas. Son propuestas de medición, cuantitativas, centradas en la función de IC, y en beneficios económicos (Jaworski y Wee, 1993; Simon y Blixt (1995), Herring, 1996). Posteriormente las propuestas de indicadores son más elaboradas y se centran más en aspectos organizativos y procesos del ciclo de la IC. Un ejemplo es la propuesta de Simon (1998). Otros están centrados en el retorno de la inversión (Kilmetz y Bridge, 1999; Davison; 2001) o en la habilidad de la organización a emplear la 
IC (Williams y Williams, 2004). Últimamente, se han desarrollado modelos tomando como base la metodología de los cuadros de mando, alineados con los objetivos de la organización, porque integran mediciones cuantitativas y cualitativas de las operaciones de IC (Buchda, 2007; Lönnqvist y Pirttimäki, 2006; Pirttimäki, Lönnqvist y Karjaluoto, 2006; Viscount, 2002; Fleisher y Blenkhorn, 2001; Herring, 1996).

\subsection{Vías de comunicación entre expertos y consolidación de la disciplina IC}

Para que un ámbito de investigación y prácticas se conviertan en disciplina hacen falta una serie de requisitos. Tres de ellos son: a) existencia de paradigmas comunes que evalúen la validez y fiabilidad de las prescripciones hechas en la literatura (Kuhn, 2006); b) la formación de grupos o redes de expertos que configuren la comunidad científica de IC, y c) la existencia de espacios de comunicación: foros, congresos y publicaciones (Vanderstraeten, 2010).

En la literatura apreciamos conciencia de la necesidad de avanzar en la construcción de un corpus teórico y un paradigma común (Saayman et al. 2008; Antia y Hesford, 2007; Calof, 2006; Brouard, 2006; Bergeron y Hiller, 2001; Serpa, 2000). Desde la ciencia de la información y la documentación se apunta la incorporación de marcos epistemológicos propios de la ciencia de la información, que contemple las necesidades y los usos de la información desde el punto de vista de los comportamientos informacionales. Estos aspectos quedan fuera del paradigma actual predominante, procedente del campo de la administración de empresa (Bergeron y Hiller, 2002; Serpa, 2000).

En contraste a la reflexión sobre la construcción del corpus de conocimiento, constatamos ausencia de una reflexión entorno a los otros dos temas: formación de grupos y espacios de comunicación. No obstante, la revisión de nombres de autores, de congresos y de publicaciones arroja algunos datos.

En relación a grupos o redes de expertos, una revisión de la literatura revela la existencia de grupos de investigación asociados a distintas disciplinas, en paralelo a las universidades que han implementado la formación específica en IC. No obstante, detectamos falta de grupos de investigación interdisciplinares, a pesar de que haya consenso en señalar la IC como interdisciplinar.

Esta separación disciplinar al abordar la IC se refleja también en las publicaciones y la organización de congresos. A nivel académico faltan publicaciones y congresos con factor de impacto, y asociaciones consolidadas de investigadores. No obstante la IC cuenta con espacios para que la comunidad científica se comunique de manera presencial, siendo aún predominantes los congresos con predominio de temas tecnológicos. Estos espacios son:

- Coloquio Veille Strategique Scientifique et Technologique (VSST) organizado por la Universidad Paul Sabatier (Toulouse 3) es el que cuenta con más tradición. Se celebra desde 1995 cada 3 años. Este coloquio, aunque desde sus inicios tiene un predominio de temas tecnológicos, en los últimos años aglutina también a expertos en IC que investigan otros temas vinculados con la IC. De hecho, a partir de 2012, debido a la alta afluencia, han establecido unos seminarios dentro del intervalo de tres años (VSST, 2012). Ello en parte es consecuencia de la inexistencia de un Congreso específico de IC que englobe por definición todas las temáticas de IC.

- European Competitive Intelligence Symposium (ECIS), cuya $3^{a}$ edición fue en 2009 con una periodicidad de 3 años, y de momento consta sin continuidad.

- Systèmes d'Information et Intelligence Economique (SIIE) que a fecha 2012 ya va por la $5^{a}$ edición y está orientado a temas tecnológicos relacionados con la IC (SIIE, 2012).

- Technological Innovation and Competitive Technical Intelligence (ITICTI), impulsado por la Universidad de Pekin, y focalizado en aplicaciones y mejoras prácticas de inteligencia tecnológica competitiva (ITICTI, 2010).

- EBRF (Research Forum to Understand Business in Knowledge Society) es un congreso mixto de investigadores y profesionales con sede en Finlandia, e impulsado sobre todo por diversas universidades del país, que pone énfasis en el campo de ciencias empresariales.

En cambio, a nivel profesional sí que hay una comunidad consolidada, que cuenta con su asociación - Strategic and Competitive Intelligence Professionals (SCIP) - con organizaciones afiliadas de países de distintos continentes, su revista -Competitive Intelligence Magazine, y celebración de varios congresos anuales Summit- en los distintos continentes. Este espacio al tener una orientación profesional sirve para debatir prácticas entre profesionales, siendo difícil introducir ponencias y debates de ámbito académico referidos a los paradigmas de 
la disciplina (metodologías, definición del problema o marcos teóricos de análisis) (SCIP, 2012). Aparte del SCIP, hay otras iniciativas de congresos y conferencias en el ámbito profesional. Es el caso del International Competitive Intelligence Conference (ICl Konferenz).

Recientemente (2011) ha surgido una nueva revista peer reviewed - Journal of Intelligence Studies in Business (JISIB) - que tiene por objetivo aglutinar expertos de la IC del ámbito académico, en las áreas de Competitive Intelligence, Business Intelligence, Market Intelligence, Scientific y Technical Intelligence and Geoeconomics. Ha sido fruto de la reflexión y de la inquietud, manifestadas en un foro de IC, de un grupo de académicos para responder a las necesidades de disponer de espacios con factor de impacto para desarrollar sus carreras profesionales (Competitive Intelligence, 2009).

\section{Discusión y conclusiones}

De entre los muchos temas objeto de debate en la literatura sobre inteligencia competitiva (IC), destacamos tres: a) terminología empleada para referirse a las prácticas, b) definición del término, y c) construcción del corpus teórico o de conocimiento, vinculado a su adscripción a un área de conocimiento. A estos temas añadimos uno más para el debate, la necesidad de consolidar espacios específicos de IC para una fluida comunicación entre los académicos de IC.

En relación al primer tema de debate, la proliferación de definiciones y la diversidad terminológica, en parte, son consecuencia de las aportaciones procedentes de diferentes disciplinas, de las prácticas y de la necesidad de diferenciar IC de otras prácticas, así como de acotar de manera clara cuál es el alcance y cuáles son sus limitaciones. Estos hechos son propios de un campo que todavía se está consolidando, partiendo de aportaciones de varias disciplinas (Larivet, 2009; Brody, 2008; Saayman et al. 2008; Calof y Wright 2008; Fleisher, Wright y Tindale, 2007; Calof, 2006; Dishman, Fleisher, Knip, 2003; Ganesh, Miree y Prescott, 2003). De hecho, la dispersión terminológica, junto a la dispersión de publicaciones que recogen trabajos en IC, dificulta la localización del conocimiento ya producido en la IC, $\mathrm{y}$, por tanto la localización de los expertos, y la comunicación entre la comunidad científica para crear redes de conocimiento. La consecuencia puede ser lentitud en la consolidación del corpus teórico, y los trabajos empíricos.

En relación al segundo tema de debate, la construcción de un corpus de conocimiento, en la literatura apreciamos conciencia de la necesi- dad de avanzar en la construcción de un corpus teórico y un paradigma común (Saayman et al. 2008; Antia y Hesford, 2007; Calof, 2006; Brouard, 2006; Bergeron y Hiller, 2002; Serpa, 2000). Sin embargo para avanzar en estas líneas es preciso apostar por el trabajo conjunto multidisciplinar, y fomentar el debate en torno a las metodologías adecuadas a los problemas planteados en IC, ya que aún hay influencia de las tradiciones metodológicas de cada disciplina, olvidando a veces el carácter multidisciplinar del objeto de análisis, que pueda requerir tratamientos distintos al de la disciplina de origen del investigador (Serpa, 2000). Además de seguir trabajando en la definición de los métodos de investigación, otras líneas de trabajo son: a) factores que influyen las prácticas de IC incorporándolas además a la función y ciclo de inteligencia; b) peculiaridades de las prácticas en distintos sectores y sus entornos, para estudiar cómo la IC puede contribuir en la generación de valor e innovación, que repercutan en el desarrollo del área donde las organizaciones desarrollan su actividad.

Por ultimo, el cuarto tema de debate, y el que requiere de un mayor impulso, es la creación de espacios específicos para la comunidad científica especializada en IC. Contar con dichos espacios permite una comunicación más fluida entre ellos, y definir líneas de investigación en las que trabajar de manera conjunta. Esto a su vez facilitará superar la dispersión de publicaciones, que dificultan la localización del conocimiento ya generado en el campo de la IC.

Si la emergencia de una disciplina está conectada con la conformación de paradigmas comunes (Kuhn, 2006), la formación de grupos o redes de especialistas, el surgimiento de comunidades de científicos y el establecimiento de publicaciones especializadas que faciliten la formación de redes (Vanderstraeten 2010), podemos concluir que la consolidación de la disciplina lleva ya un largo camino recorrido, y está en vías de consolidarse, no obstante aún queda un trecho del camino por recorrer.

\section{Referencias}

Aguilar, F. J. (1967). Scanning the business environment. Mcmillan: New York.

Alaqui, M. (2010). Use of "Territorial Intelligence" in the development of industrial clusters in Morocco case of the firts seadfood processing cluster in Morocco. Edinburg Napier University (TesiS Doctoral)

Ansoff, H. I. (1975). Managing Strategic Surprise by Response to Weak Signals. // California Management Review. 18:2, 21-33.

Antia, K.; Hesford, J. (2007). A process-oriented view of competitive intelligence and its impact on organizational 
performance. // Journal of Competitive Intelligence and Management. 4:1, 5-33.

Antia, K.; Hesford, J. (2007). A process-oriented view of competitive intelligence and its impact on organizational performance. // Journal of Competitive Intelligence and Management. 4:1, 5-33.

Babbar, S.; Rai, A. (1993). Competitive intelligence for international business. // Long Range Planning. 26:3, 103113

Bégin, L.; Deschamps, J.; Madinier, H. (2007). Une approche interdisciplinaire d'intelligence économique. An interdisciplinary approach of competitive intelligence. Cahier de recherche $n^{\circ}$ HES-SO/HEG-GE/C-07/4/4$\mathrm{CH}$.

Bensoussan, B.; Densham, E. (2004). Australian Cl Practices: a comparaison with the U.S. // Journal of Competitive Intelligence and Management. 2:3, 1-9.

Bergeron, P. (2000). Regional business intelligence: the view from Canada. // Journal of Information Science. 26:3, 153-160.

Bergeron, P; Hiller, C.A. (2002) Competitive Intelligence. // Annual Review of Information Science and Technology. . 36:1, 353-390.

Bertacchini, Y.; Girardot, J.J. (2006). De l'intelligence territoriale: théorie, posture, hypothèses, définitions. En Ve. Colloque TIC\& territoire: quels développements? Université de Franche Comté, Besançon, 9-10 juin 2006.

Blenkhorn, D. L.; Fleisher, C. S. (2007). Performance assessment in competitive intelligence: an exploration, synthesis, and research agenda. // Journal of Competitive intelligence and management. 4:2, 4-22.

Bose, R. (2008). Competitive intelligence process and tools for intelligence analysis. // Industrial Management \& Data Systems. 108:4, 510-528.

Bourret, C. (2009). «L'Intelligence territoriale: un nouvel état d'esprit?» // Colloque INTD /ENACT Les nouveaux territoires de 'information et de la documentation dans les collectivités territoriales (Nancy, 10 de noviembre de 2009).

Brody, R. (2008). Issues in defining competitive intelligence: an exploration. // Journal of Competitive Intelligence and Management 4:3, 3-16.

Brouard, F. (2006). Development of an expert system on environmental scanning practices in SME: tools as a research program. // Journal of Competitive Intelligence and Management. 3:4, 37-58.

Brouard, F.; Larivet, S.; Sakka, O. (2010). Social Entrepreneur: Definitions and Boundaries. // Canadian Journal of Nonprofit and Social Economy Research. 1:1, 46-64.

Buchda, S. (2007). Rulers for business intelligence and competitive intelligence: an overview and evaluation of measurements approaches. // Journal of Competitive Intelligence and Management. 4:2, 23-54.

Calof, J. (2006). The SCIP06 Academic Program-Reporting on the State of the Art. // Journal of Competitive Intelligence and Management, vol. 3, $n^{\circ} 4$, p. 5-13.

Calof, J. L.; Skinner, B. (1999). Creating an intelligence society: the role of government in competitive intelligence. What's happening in Canada?. // Competitive Intelligence Magazine. 2:2, 20-23.

Calof, J.; Brouard, F. (2004). Competitive Intelligence in Canada. // Journal of Competitive Intelligence and Management. 2:2, 1-21.

Calof, J.; Wright, S. (2008). Competitive intelligence: a practitioner, academic and inter- disciplinary perspective. // European Journal of Marketing. 42(7/8) 717-730.
Calof, J.L.; Dishman, P. (2002). The intelligence process: front-end to strategic planning ( Working paper). Ottawa: University of Ottawa.

Carayon, Bernard (2003). Intelligence économique, compétitivité et cohésion sociale: rapport au Premier ministre. Paris: La Documentation française, 2003.

Cartwright, D. L.; Boughton, P. D.; Miller, S. W. (1995). Competitive intelligence systems: relationships to strategic orientation and perceives usefulness. // Journal of Managerial Issues. 7:4, 420-434.

Choo, C. W. (2001). Environmental scanning as information seeking and organizational learning. // Information Research. 7:1,.. http://informationr.net/ir/7-1/paper112.html (20 de mayo de 2011).

Choo, C. W. (2002) Information management for the Intelligent Organization: the art of scanning environment. Medford: Asis\&T, Information Today.

Choo, C. W; Bergeron, P.; Detlor, B.; Heaton, L. (2008). Information Culture and Information Use: An exploratory study of three organizations. Journal of the American Society for Information Science and Technology, v. 59, $\mathrm{n}^{\circ} 5$, p. 792-804.

Choo, Ch. W.; Auster, E. (1993). Environmental scanning: acquisition and use of information by managers. // Annual Review of Information Science and Technology. 28 , 279-314.

Comai, A.; Tena Millán, J. (2005). La intel·ligència competitiva a Espanya: desenvolupament actual i perspectives futures. // Bibliodoc. 32, 31-45.

Competitive Intelligence (2009). Interest in starting an onlineonly academic $\mathrm{Cl}$ journal? // Competitive Intelligence: Tactical, Operational \& Strategic Analysis of Markets, Competitors \& Industries. http://competitiveintelligence.ning.com/forum/topics/inter est-in-startingan?xg_source=activity\&id=2036441\%3ATopic\%3A2216 8\&page $=2 \#$ comments (Mayo 2012)

Correia, Zita; Wilson, T.D. (1997). Scanning the business environment for information: a grounded theory approach. I/ Information Research. 2:4, http://informationr.net/ir/2-4/paper21.html (30 de junio de 2011).

Cronin, B.; Davenport, E. (1993). Social Intelligence. // Annual Review of Information Science and Technology. 28:1, 23-44.

Culver, Martha (2006). Using tactical intelligence to help inform strategy. // Strategy \& Leadership. 34:6, 17-23.

Daft, R.; Weick, K. E. (1884). Towards a model of organizations as interpretation Systems. // Academy of Management Review. 9:2, 284-295.

Dashman, L. (1998). The value of an in-house competitive intelligence department: a business plan approach. // Competitive Intelligence Review. 9:2, 10-16.

Davison, L. (2001). Measuring competitive intelligenc efectiveness: insights from the advertising industry. // Competitive Intelligence Review. 12:4, 25-38.

De Pelsmacker, P.; Muller, M-L., Viviers, W.; Cuyvers, L.; Jegers, M. (2005). Competitive intelligence practices of South African and Belgian exporters. // Marketing Intelligence Planning. 23:6, 606-620.

Diffenbach, J. (1983). Corporate environmental analysis in larger US corporations. // Long Range Planning. 16:3, 107-16.

Dishman, P. L.; Pearson, T. (2003). Assessing intelligence as learning within an industrial marketing group: a pilot study. // Industrial Marketing Management. 32:7, 615620. 
Dishman, P.; Fleisher, C.; Knip, V. (2003). Chronological and Categorized Bibliography of Key Competitive Intelligence Scholarship: Part 1 (1977-present). // Journal of Competitive Intelligence and Management. 1:1, 13-79.

Dishman, Paul L.; Calof, Jonathan L. (2008). Competitive intelligence: a multiphasic precedent to marketing strategy. // European Journal of Marketing. 42(7/8) 766-785.

Ebrahimi, B. P. (2000). Perceived strategic uncertainty and environmental scanning. Behavior of Hong Kong Chinese executives. // Journal of Business Research. $49,: 1,67-77$

EBRF http://www.ebrf.fi

ECIS (2009) http://www.atelis.org/Version_ang/pages/evene ments_3colloque_europeen.htm

Escorsa, P.; Maspons, R. (2001). De la vigilancia tecnológica a la Inteligencia Competitiva. Madrid: Prentice-Hall.

Fahey, L.; King, W.; Narayanan, V. K. (1981). Environmental scanning and forecasting in strategic planning-the state of the art. // Long Range Planning. 14, 32-39.

Fink, A.; Schlake, O. (2000) Scenario management-An approach for strategic foresight. // Competitive Intelligence Review. 11:1, 37-45.

Fleisher, C. S.; Bensoussan, B. E. (2008). Business and competitive analysis: effective application of new and classic methods. Upper Saddle River, New Jersey: Pearson Education, Inc.

Fleisher, C. S.; Blenkhorn, D. L. (ed.) (2001). Managing Frontiers in Competitive Intelligence. Westport, London: Quorum Books.

Fleisher, C.; Knip, V.; Dishman, P. (2003). Chronological and categorized bibliography of key competitive intelligence scholarship: Part 2 (1990-1996). // Journal of Competitive Intelligence and Management. 1:2, 11-86.

Fleisher, C.; Wright, S.; Tindale, R. (2007). Bibliography and assessment of key competitive intelligence scholarship: part 4 (2003-2006). // Journal of Competitive Intelligence and Management. 4:1, 34-107.

Fox, J.A.; Brown, L.D. (Eds.) (1998). The Struggle for Accountability: The World Bank, NGOs and Grassroots Movements. Cambridge: MIT Press.

Frion, P. (2009). What information behavior can offer to competitive intelligence?. http://s244543015.onlinehome. fr/ciworldwide/wp-content/uploads/2010/01/frion_info_be havior.pdf (30 de junio de 2011)

Frion, P.; Samier, H. (2009). Looking for Information: a New Approach to consider Efficiency and Effectiveness. En ECIS The 3rd European Competitive Intelligence Symposium. Suecia: Junio 11-12, Stockholm.

Ganesh, U.; Miree, C. E.; Prescott, J. (2003). Competitive intelligence field research: moving the Field forward by setting a research agenda. // Journal of Competitive Intelligence and Management.. 1:1, 1-12.

Garcia Alsina, M. (2011). Contribución de la inteligencia competitiva en el proceso de adaptación al EEES: el caso de las universidades españolas. Universitat Oberta de Catalunya (Tesis doctoral).

Ghoshal, S.; Kim, S. K. (1986). Building effective intelligence systems for competitive advantage, // Sloan Management Review. 28:1, 49-58.

GIA-Global Intelligence Alliance (2005). Competitive Intelligence in Large Companies. // Global Study. GIA White Paper, $n^{\circ} 4$.

GIA-Global Intelligence Alliance (2007). Market Intelligence in Large Companies. Global Study. // GIA White Paper,

Gieskes, H. (2000). Competitive intelligence at Lexis-Nexis. // Competitive Intelligence Review. 11:2, 4-11.
González Galvez, P. (2012). Xarxes socials com a instrument analític de suport a les funcions d'intel-ligència de les organitzacions. Estudi de cas de les direccions d'infermeria". Universitat de Barcelona: Tesis Doctoral (sin publicar)

Hair, Joe F. (2007). Knowledge creation in marketing: the role of predictive analytics. // European Business Review. 19:4, 303-315.

Hedin, H. (2004). Evolution of Competitive Intelligence in Sweden. // Journal of Competitive Intelligence and Management. 2:3, 56-75.

Heinrichs, J.H.; Lim, J.S. (2005). Model for organizational knowledge creation and strategic use of information. // Journal of the American Society for Information Science and Technology. 56:6, 620-629.

Heppes, D.; Du Toit, A. (2009). Level of maturity of the competitive intelligence function: case study of a retail bank in South Africa. // Aslib Proceedings: New Information Perspectives. 61:1, 48-66.

Herbaux, Ph.; Bertacchini, Y. (2007). «Part du construit semiologique en intelligence territoriale». // Vle Colloque International "TIC \& Territoire: quels développements?" (Lyon, 14 y 15 de junio de 2007).

Herring, J. P. (2007). How much is your competitive intelligence worth? // Competitive Intelligence Magazine, vol. $10, n^{\circ} 2$, p. 23-26.

Herring, J.P. (1996). Measuring the effectiveness of competitive intelligence: Assessing and communicating Cl's value to your organization. Alexandria, VA: Society of Competitive Intelligence Professionals.

Hesford, J. W. (2008). An empirical investigation of accounting information use in competitive intelligence. // Journal of Competitive Intelligence and Management,.4:3, 17-49.

Hirvensalo, I. (2004). Competitive intelligence in Finland. // Journal of Competitive Intelligence and Management. 2:2, 22-37.

Hofstede, Geert (2001). Culture's consequences. Thousand Oaks, London, New Delhi: Sage Publications.

ICl. http://conference.competitive-intelligence.com/index.sh tml

Ikeya, N; Ishikawa, K. (2001). The japanese intelligence culture. // Competitive Intelligence Review. 12:4, 51-56.

ITICTI

http://english.pku.edu.cn/News_Events/News/Global/756 8.htm

Jain, C.; Singhvi, S. S. (1977). Environmental forecasting and non-profit professional organizations. // Long Range Planning. 10:3, 50-58.

Jain, S. (1984). Environmental scanning in US Corporations. // Long Range Planning. 17:2, 117-128.

Jaworski, B.; Macinnins, D.; Kholi, A. (2002). Generating Competitive Intelligence in Organizations. // Journal of Market-Focused Management. 5:4, 279-307.

Jaworski, Bernard J.; KOHLI, Ajay, K. (1993). Market orientation: antecedents and consequences. // Journal of Marketing. 57:3, 53-70.

JISIB (2012) https://ojs.hh.se/index.php/JISIB/index

Juhari, Ariff; Stephens, Derek (2006). Tracing the origins of competitive intelligence throughout history // Journal of Competitive Intelligence and Management. 3:4, 61- 82.

Kamoun-Chouk, S. (2007). Environmental scanning in Tunisian Manufacturing SMEs. // Journal of Information \& Knowledge Management. 6:1, 57-67.

Kilmetz, D.; Bridge, R. S. (1999). Gauging the returns on investments in Competitive intelligence: a three-step analysis for executive decision makers. // Competitive Intelligence Review. 10:1, 4-11. 
Kim, K.; Kim, S. (2004). Competitive intelligence in Korea. // Journal of Competitive Intelligence and Management. $2: 3,10-25$.

King, W. R.; Cleland, D. I. (1974). Environmental Information Systems for Strategic Marketing Planning. // Journal of Marketing. 38:4, 35-40.

Knip, V. (2006). Culture counts! Teaching business students why and how to leverage cultural analysis to optimize global macro-environmental scanning. // Journal of Competitive Intelligence and Management. 3:4, 85-110.

Knip, V.; Dishman, P.; Fleisher, C. (2003). Chronological and categorized bibliography of key competitive intelligence scholarship: part 3 (the earlist writings-1989). // Journal of Competitive Intelligence and Management. 1:3, 10-79.

Kotorov, R. P. (2001). The strategy wheel: a method for analysis and benchmarking for competitive strategy. // Competitive Intelligence Review. 12:3, 21-30.

Kourteli, L. ( 2005). Scanning the business external environment for information: evidence from Greece. Information Research.11:1, paper 242. http://InformationR.net/ir/11-1/paper242.html. (30 de junio de 2011)

Kuhn, T. S. (2006). La Estructura de las revoluciones científicas. Madrid (etc.): Fondo de Cultura Económica, 2006.

Lackman, L.; Saban, K.; Lanasa, J. M. (2000). Organizing the Competitive Intelligence Function: a benchmarking study. // Competitive Intelligence Review. 11:1, 17-27.

Larivet, S. (2007). Panorama des practiques d'intelligence économique europénnes. // Les cahiers de recherche, 8, 33-50.

Larivet, S. (2009). "Economique inteligence in Small and Medium Businesses in France: a survey". // Hoppe, M.; Hamrefors, S.; Silen, K. ECIS 2009 Third European Competitive Intelligence Symposium: Competitive intelligence: competing, consuming and collaborating in a flat world (Stockolm, 11 y 12 de junio de 2009).

Larivet, S. (2009). Intelligence Économique: Enquête dans 100 PME. Paris: L'Harmattan.

Liu, Chun-Hsien.; Wang, Chu-Ching (2008). Forecast competitior service strategy with service taxonomy and $\mathrm{Cl}$ data. // European Journal of Marketing. 42(7/8) 746-766.

Lönnqvist, A.; Pirttimäki, V. (2006). The measurement of Business Intelligence. // Information Systems Management. 23:1, 32-40.

Marin, J.; Poulter, A. (2004). Dissemination of Competitive Intelligence. // Journal of Information Science. 30:2, 165180.

Marín-Llanes,L.; Carro-Cartaya, J.; Espín-Andrade, R. (2001). Information analysis techniques for the competitive intelligence process. // Competitive Intelligence Review. 12:1, 32-40.

Martre 1994. Intelligence économique et stratégie des entreprises: Rapport Marte. Commissariat Général du Plan. Paris: La Documentation Française.

McGonagle, J. (2007). An examination of the "classic" Cl model. // Journal of Competitive Intelligence and Management. 4:2, 71-86

McGonagle, J.; Vella, C. M. (2003). The managers's guide to competitive intelligence. Westport, Connecticut: Praeger Publishers.

Michaeli, R. (2004). Competitive Inteligence in Germany. // Journal of Competitive Intelligence and Management. 2:4, 1-6.

Michaeli, R. (2006). Competitive intelligence: strategische Wettbewerbsvorteile erzielen durch systematische Konkurrenz-, Markt-und Technologieanalysen. Berlin, Heidelberg: Springer.
Miller, J.P. (2001) Millennium intelligence: understanding and conducting competitive intelligence in the digital age. Medford, New York: CyberAge books.

Miller, K. D. (2002). Competitive strategies of religious organizations. // Strategic Management Journal. 23:5, 435456

Olamade, Olumuyiwa O.; Oyebisi, T. O.; Egbetokun, A. A.; Adebowale, Boladale (2011). Environmental scanning strategy of manufacturing companies in southwestern Nigeria. // Technology analysis \& strategic management. 23:4, $367-381$.

Ortoll, E; García, M. (2008). La Intel-ligència Competitiva. Barcelona: Editorial UOC. Col. Vull Saber.

Parker, K. R.; Nitse, P.S.; Davey, B. (2008). History of Computing Education Trends: the emergence of competitive intelligence. // IFIO International Federation for Information Processing. 269; History of Computing and Education 3, 113-127. Boston: Springer.

Peyrot, M.; Childs, N. ; Van Doren, D.; Kathleen, A. (2002). An empirically based model of competitor intelligence use. // Journal of Business Research. 55, 747-758.

Pirttimäki, V. (2007). Comparative Study and Analysis of the intelligence activites of large Finnish companies. // Journal of Competitive Intelligence and Management. 4:1, 147-170.

Pirttimäki, V.; Lönnqvist, A.; Karjaluoto, A (2006). Measurement of Business Intelligence in a Finnish Telecommunications Company. // The Electronic Journal of Knowledge Management. 4:1, 83-90.

Portail de la IE, 2010 http://www.portail-ie.fr/organisationsde-ie/ (14 de mayo de 2011).

Porter, M. (1987). Competitive Strategy. New York: Free Press.

Prescott, J. E. (1999) The evolution of Competitive Intelligence: designing a process for action. // APMP, 37-51.

Rouach, D., Santi, P. (2001). Competitive Intelligence adds value: five intelligence attitudes. // European Management Journal. 19:5, 552-559.

Saayman, A; Pienaar, J.; Pelsmacker, P.; Viviers, W.; Cuyvers, L.; Muller, M. L.; Jegers, M. (2008). Competitive intelligence: construct exploration, validation and equivalence. // Aslib Proceedings: New Information Perspectives. 60:4, 383-411.

Saayman, Andrea; Cuyvers, Ludo; Jegers, Marc (2005). Competitive intelligence practices of South African and Belgian exporters. // Marketing intelligence \& planning. $23: 6,606-620$

SAWKA, K. (2000). Are We Valuable? // Competitive Intelligence Magazine. 3:2, 53-54.

SCIP (2012) http://www.scip.org/

SERPA, L. F. (2000). Epistemological assessment of current business intelligence archetypes. // Competitive Intelligence Review. 11:4, 88-101.

SIIE (2012) http://siie2011.loria.fr/

Simon, N. J. (1999). The effects of organizational culture on the $\mathrm{Cl}$ process. // Competitive Intelligence Review. 10:1, p. 62-70.

Simon, Neil; Blixt, Albert (1995). Emerging issues in competitive intelligence, 1994. // Competitive Intelligence Review. 6:2, 42-56.

Smith, J.R., Wright, S. And Pickton, D.W. (2010) Competitive Intelligence as Public Policy in France: Making a Difference in the SME Sector. // Academy of Marketing Conference, Competitive Intelligence, Analysis \& Strategy Track. Coventry, UK 
Solberg Søilen, K. (2005). Introduction to private and public intelligence. The Swedish School of Competitive Intelligence. Lund: Studentlitteratur.

Stankeviciute, J.; Oržekauskas, P.; Jucevicius, R. (2004). Competitive intelligence in Lithuania. // Journal of Competitive Intelligence and Management. 2:4, 32-41.

Thomas, J. H. (2001). Business Intelligence: Why? // eAI Journal. (Julio 2001) 47-49.

Thomas, P. S. (1974). Environmental analysis for corporate planning. // Business Horizons. 17:5, 27-38.

Vanderstraeten, R. (2010). Scientific Communications: Sociology Journals and Publications Practices. // Sociology. 44:3,559-576.

Viscount, C. (2002). Using the balanced scorecard process for evaluating the contribution of a competitive intelligence effort. Chapel Hill: Faculty of the School of Information and Library Science of the University of North Carolina. (Trabajo de Master of Science in Information Science). http://www.ils.unc.edu/MSpapers/2770.pdf (30 de junio de 2011).

Viviers, W.; Muller, M. L. (2004). The evolution of competitive intelligence in South Africa Early 1980s-2003. // Journal of Competitive Intelligence and Management. 2:2, 53-67

Viviers, W.; Saayman, A.; Muller, M. L. (2005). Enhancing a competitive intelligence culture in South Africa. // International Journal of Social Economics. 32:7, 576-589.

VSST 2012. http://atlas.irit.fr/

Vuori, V. (2006). Methods of defining business information needs. http://www.ebrc.fi/kuvat/Vuori_paper.pdf . (30 junio de 2011)
Wagner, R. B. (2003). Can Competitive Intelligence be Effectively applied to Public Sector Organizations?. I/ Fleisher, C. S.; Blenkhorn, D. L. (eds.). Controversies in Competitive Intelligence: the enduring issues. Westport, CT: Praeger Books. p. 70-82.

Weerawardena, J.; Mcdonald, R. E.; Mort, G. S. (2010). Sustainability of nonprofit organizations: an empirical investigation. // Journal of World Business. 45, 346-356.

Weiss, A. (2002). A brief guide to competitive intelligence: how to gather and use information on competitors. II Business Information Review. 19:2, 39-47.

Williams, S., Williams, N. (2004), Capturing ROI through Business-Centric BI Development Methods. // DM Review, August 2004

Wright, S.; Calof, J. L. (2006). The quest for competitive, business and marketing intelligence: a country comparison of current practices. // European Journal of Marketing. 40(5/6) 453-465.

Wright, S.; Pickton, D.W.; Callow, J. (2002). Competitive intelligence in UK firms: a typology. // Marketing Intelligence \& Planning. 20:7, 349-360.

Wright, S; Badr, A.; Weiss, A.; Pickton, D. (2004) Competitive Intelligence through UK Eyes. // Journal of Competitive Intelligence and Management. 2:2, 68-87.

Zhang, X.; Majid, S.; Foo, S. (2010). Environmental scanning: An application of information literacy skills at the workplace. // Journal of Information Science. 36:6, 719732

Enviado: 2012-05-15

Aceptado: 2012-07-01. 